\title{
Clinical Trial Participant Willingness and Influencing Factors Study of Patients with Inflammatory Bowel Disease
}

\author{
Ni Ding, Huiping Chen, Qianqian Ji, Mengting Hu, Xiang Gao* \\ Department of Gastroenterology, The Sixth Affiliated Hospital of Sun Yat-sen University, Guangzhou, China \\ Email: ^dingn8@mail.sysu.edu.cn
}

How to cite this paper: Ding, N., Chen, H.P., Ji, Q.Q., Hu, M.T. and Gao, X. (2021) Clinical Trial Participant Willingness and Influencing Factors Study of Patients with Inflammatory Bowel Disease. Open Journal of Nursing, 11, 676-681.

https://doi.org/10.4236/ojn.2021.118057

Received: July 20, 2021

Accepted: August 17, 2021

Published: August 20, 2021

Copyright (อ 2021 by author(s) and Scientific Research Publishing Inc. This work is licensed under the Creative Commons Attribution International License (CC BY 4.0).

http://creativecommons.org/licenses/by/4.0/

\section{(c) (i) Open Access}

\begin{abstract}
Objective: The study was conducted to understand the situation of patients with inflammatory bowel disease (IBD) to participant clinical trials and to analyze the factors affecting the clinical trial participation of patients with IBD. A clinical experiment guidance will be proved by this study to maximized the benefits to patients and to help the clinical trial to conduct successfully. Method: An anonymous questionnaire was designed and was administrated to the patients with IBD who were randomly delivered in the inpatient or outpatient departments. The survey result was analyzed. Result: Total 372 available questionnaires were returned. Among these patients, 26.3\% patients with IBD indicated willingness to participate, $57.3 \%$ indicated a situation dependence, and $41.04 \%$ indicated unwillingness. Among the potential factors that may influence the patient's willingness to participate the clinical, trusted physician's recommendation, no proved drugs to use and accessing to free medication to release financial burden were statistically significant. Conclusion: The overall willingness of IBD patients to participate in drug clinical trials is not high. Among the patients who are willing to participate in clinical trials, the main reasons for their participation are that they trust doctors' recommendation, can get free medication and examination, and can reduce the economic burden. Efficacy and safety were the main influencing factors in patients who were case-dependent and unwilling to participate in clinical trials.
\end{abstract}

\section{Keywords}

Inflammatory Bowel Disease (IBD), Drug Clinical Trials, Influencing Factors, New Drugs, Research Nurses 


\section{Introduction}

Inflammatory bowel disease (IBD) is a group of chronic and non-characteristic intestinal inflammatory diseases with unclear etiology, including ulcerative colitis (UC) and Crohn's disease. Population of patients with IBD has been increased significantly in the last 20 years. The population of IBD patients is estimated to reach 1.5 million patients in China by 2025 [1]. IBD is currently not curable and patients require long-term medication to maintain remission. Commonly used traditional drugs include amino salicylic acid, immunomodulatory drugs such as thiopurines and hormones [2]. However, the therapeutic efficacy of these drugs is limited with high incidence of adverse effects. With the development of immunology, the very first biologic agent, infliximab is available. The proven of infliximab to treat IBD significantly increase the remission rate of IBD. However, up to $20 \%$ of patients suffer from secondary treatment failure with infliximab every year [3] [4]. Therefore, there is still a blank for the discovery of new drugs in IBD. In recent years, with the increasing understanding of the etiology, several new drugs have already initiated global or national clinical trials. These trials will gradually make up for the lack of currently available drug therapy and provide more treat options for patients with IBD. Clinical trials refers to the systematic research, what is the drug in the body to confirm and found that the clinical pharmacology experimental drug, adverse reactions, absorption, distribution, metabolism and excretion, to determine the safety and efficacy of drugs, the subjects' rights and interests and the safety is the primary consideration, only can be expected to benefit is greater than the risk [5]. Clinical trial is the essential stage for new drugs to apply clinically. It is the only way for translational medicine, and it is of great significance for drug research and development and improvement of medical level. In recent years, with the support of national policies on new drug development, the number of clinical trials in China has grown rapidly and become an important part of the work of hospital clinicians and research nurses [6] [7]. Several studies have shown that recruiting subjects to participate the clinical trial is a common problem, and is also one of the important reasons for the slow progress of new drug invention [8]. Previous surveys that conducts in China on patients with cancer have showed that the public's awareness of drug clinical trials are limited. In addition, it is also difficult for the patients to access the relevant information. Both the limitation and difficulties were the main reasons for the low participation. The clinical trials in IBD is increasing rapidly in recent years. The same low willingness to participant issue has also arisen in IBD field. Therefore, it is necessary to analyze the willingness of patients with IBD and the factors that may influence the willingness, to prove the evidence for the conduction of clinical trials in IBD.

\section{Participants and Methods}

Participants: Patients with IBD who were having subsequent visit to the department of inflammatory bowel disease outpatient service and hospitalized in 
the Department of Gastroenterology of the Sixth Affiliated Hospital of Sun Yat-sen University from May 2021 to August 2021 were selected. Patients should meet following inclusion and exclusion criteria: Inclusion: 1) Diagnose with IBD. 2) >18 years old. 3) No other chronic disease or mental illness.

Exclusion: 1) The clinical diagnosis was unshaped enteritis. 2) Have a history of mental illness.

Method: Questionnaire Star electronic questionnaire survey was utilized to collect the demographic information and the factors that may influence the willingness to participant the clinical trial, the contents of the questionnaire were sorted out according to the concerns of patients when they talked about informed consent in the past.

Data Analyses: Data was recorded and managed by EpiData 3.1. SPSS 23.0 Software was utilized for multiple logistic regression analysis. The $\mathrm{P}$ value less than 050 indicates that the difference is statistically significant. Enumeration data were statistically described by frequency (percentage) and compared within groups by $\mathrm{X} 2$ test.

\section{Result}

1) Total 372 available questionnaires were returned. Among these patients, $26.3 \%$ patients with IBD indicated willingness to participate, $57.3 \%$ indicated a situation dependence, and $41.04 \%$ indicated unwillingness. The demographic characteristic was displayed in Table 1.

2) Influence factors of participant willingness

a) Influence factors analysis of willing to participant group and dependents on the situation group

Table 1. Demographic characteristic (n, \%).

\begin{tabular}{|c|c|c|c|}
\hline Item & Willing & Depends on situation & Unwilling \\
\hline Age & $28.80 \pm 12.56$ & $30.33 \pm 11.56$ & $31.50 \pm 13.21$ \\
\hline \multicolumn{4}{|l|}{ Gender } \\
\hline Male & $32(61.54)$ & $102(61.45)$ & $96(62.34)$ \\
\hline Female & $20(38.46)$ & $64(38.55)$ & $58(37.66)$ \\
\hline \multicolumn{4}{|l|}{ Education } \\
\hline Bachelor or above & $20(38.46)$ & $94(56.63)$ & $78(50.65)$ \\
\hline Under Bachelor & $32(61.54)$ & $72(43.37)$ & $76(49.35)$ \\
\hline \multicolumn{4}{|l|}{ Payment method } \\
\hline Self-paying & $40(76.92)$ & $66(39.76)$ & $70(45.45)$ \\
\hline insurance & $12(23.08)$ & $100(60.24)$ & $84(54.55)$ \\
\hline \multicolumn{4}{|l|}{ Time of IBD } \\
\hline 5 years or above & $22(42.31)$ & $98(59.04)$ & $55(35.71)$ \\
\hline Less than 5 years & $32(61.54)$ & $68(40.96)$ & 99 (64.29) \\
\hline
\end{tabular}


Table 2. The influence factors and willingness (n, \%).

\begin{tabular}{lcc}
\hline \multicolumn{1}{c}{ Reason for willing to participant } & $\begin{array}{c}\text { Willing } \\
(\mathrm{n}=52)\end{array}$ & $\begin{array}{c}\text { Dependents on the situation } \\
(\mathrm{n}=166)\end{array}$ \\
\hline Recommendation from trusted physicians & $48(92.30)$ & $139(83.73)$ \\
Free treatment and test & $88(53.01)$ \\
Currently available drugs do not control the disease & $52(94.54)$ & $104(62.65)$ \\
The most cutting-edge drugs & $22(42.3)$ & $120(72.29)$ \\
Acquire more attention from physicians & $39(75.00)$ & $89(53.61)$ \\
$\begin{array}{l}\text { Devote to the IBD medicine } \\
\text { Worry the attitude will be change if I refused to }\end{array}$ & $18(34.61)$ & $33(19.88)$ \\
attend the clinical trial & $10(19.23)$ & $22(13.25)$ \\
\hline
\end{tabular}

Note: ${ }^{*} \mathrm{P}<0.05$.

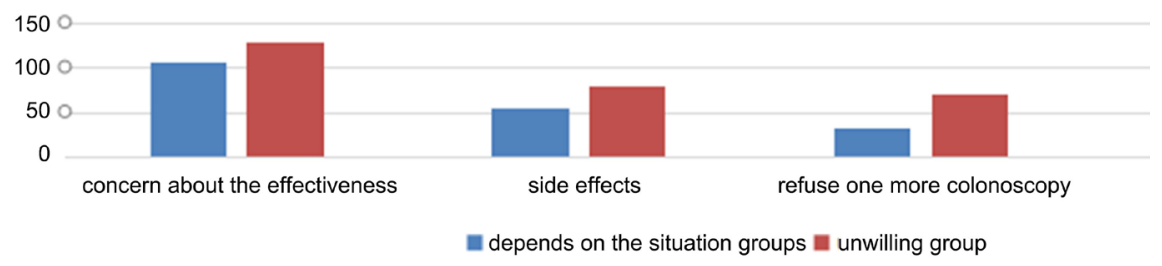

Figure 1. Influence factors analysis of dependents on the situation group and unwilling group.

Recommendation from trusted physicians, free treatment and examination, currently proved drugs cannot control the disease, not to participate in the experiment affect the attitude of doctors to themselves, the differences are statistically significant $(\mathrm{P}<0.05)$. The results are shown in Table 2 .

b) Influence factors analysis of dependents on the situation group and unwilling group

$105(63.25 \%)$ of patients in the "depends on the situation groups" and 128 $(83.12 \%)$ in the "unwilling" group are concern about the effectiveness of the new drug. In addition, 123 (74.10\%) and 117 (75.94\%) of patients in "depends on the situation" and "unwilling" group respectively are concern about the side effects of the new drug. $33(19.88 \%)$ and $69(44.81 \%)$ in "depends on the situation" group and "unwilling" group refuse to have another colonoscopy and other tests. All these differences were statistically significant $(\mathrm{P}<0.05)$ (Figure 1$).$

\section{Discussion and Conclusions}

With the deeper understanding of the pathogenesis of IBD and the rapid development of pharmaceutical technology, newly developed drugs may bring better therapeutic effect and less side effects [9]. As one of the key stages for new drugs to be marketed and widely used in clinical practice, clinical trial can not only help patients benefit from the trials to a certain extent, but also make important contributions to the improvement of medical progression. The clinical trial can accelerate the pace of new drugs to be marketed, and provide patients with more 
and better treatment options. Therefore, it is particularly important to improve and promote the active participation of patients with IBD in new drug trials.

Most of the patients with IBD are middle-aged and young patients, and their acceptance of new drugs should be relatively higher than the elder age people. Among the main factors influencing patients' participation in drug clinical trials, recommendations from trusted physicians, accessing to free drugs and tests, and reduction of economic burden are the main reasons. Inflammatory bowel disease (IBD) is a chronic disease, which requires patients to seek for treatment occasionally and encounter disease-related problems in daily life. In this process, the trust relationship with doctors is gradually established, and the recommendation of doctors becomes one of the main factors for patients to participate in clinical trials. In this way, clinicians are required to be very familiar with various new drug projects carried out by the department and to recommend appropriate items to patients who initially meet the admission and discharge standards in a timely manner. Expenses for tests and drugs related to IBD put heavy economic pressure on patients, especially those in the active stage of the disease, who may not be able to work properly. A survey shows that in the United States, patients with CD and UC will face a lifetime medical expense of $\$ 416,352$ and $\$ 195,799$ respectively, which is significantly higher than chronic diseases such as diabetes and rheumatoid arthritis [10]. However, during the period of clinical trial, not only the study drug is free, but also the tests related to the trial is free, which can relieve the medical economic pressure for patients with IBD to a certain extent.

According to patients who unwilling to participate in clinical trials or whose participant's willingness depends on situation, patients were most concerned about the efficacy and safety of the drugs when encounter the clinical trials. Due to the fact that the diagnosis and treatment of IBD fall behind in China than in Europe and the United States, many clinical trials under clinical trial in China are generally launched later than those in foreign countries. In other words, the drugs under clinical trial in China have been proved effective and safety to a certain extent at the early stage. When discussing with patients about informed consent, relevant data, drug mechanism, and whether the drug has been marketed abroad or domestically as other indications can be introduced to patients, so as to eliminate patients' concerns about the effectiveness and safety of new drugs to a certain extent. Afraid to be randomized to placebo group is also one of the reasons why patients reject to participate in the new drug program. In order to ensure the scientific design of clinical trials, many of the control groups were placebos, and patients would therefore refuse to participate because of the uncertain of the benefit. Research nurses require assisting researchers to explain to the patients, in several clinical trials, background medicines are allowed to use during the experiment. In addition, IBD is a kind of chronic diseases, the progress of the disease will generally not very quickly. In many IBD clinical trials, the length of placebo phase is only about 8 to 12 weeks. The clinical indicators of IBD will be assessed after that. The effect of the drug (new drug or placebo) will be measured. The patients with no response in the placebo group will be reattributed 
reassigned to the experiment group. In addition, the probability of being assigned to placebo group is lower. As one of the direct participants in the clinical trial, the research nurses help the researcher to popularize the knowledge to the patients, and promote the patients with IBD to participate the clinical trials. To sum up, trusting the doctor's recommendation, obtaining free medication and examination, reducing the economic burden, drug effectiveness and safety are the main factors affecting the participation of IBD patients in drug clinical trials.

\section{Fund}

Guangdong Medical Science and Technology Research Fund Project No. C2021047.

\section{Conflicts of Interest}

The authors declare no conflicts of interest regarding the publication of this paper.

\section{References}

[1] Wang, Y. and Ouyang, Q. (2007) Ulcerative Colitis in China: Retrospective Analysis of 3100 Hospitalized Patients. Journal of Gastroenterology and Hepatology, 22 1450-1455. https://doi.org/10.1111/j.1440-1746.2007.04873.x

[2] Wu, K.C., Liang, J., Ran, Z.H., et al. (2018) Consensus on Diagnosis and Treatment of Inflammatory Bowel Disease. Journal of Gastroenterology, 38, 292-311.

[3] Pouillon, L., et al. (2016) Considerations and Considerations, Challenges and Future of Anti-TNF Therapy in Treating Inflammatory Bowel Disease. Expert Opinion on Biological Therapy, 16, 1277-1290. https://doi.org/10.1080/14712598.2016.1203897

[4] Roda, G., Jharap, B., Neeraj, N. and Colombel, J.-F. (2016) Loss of Response to Anti-TNFs: Definition, Epidemiology, and Management. Clinical and Translational Gastroenterology, 7, e135.

[5] (2020) National Food and Drug Administration, National Health Commission Announcement on the Release of Drug Clinical Trial Quality Management Practice. Bulletin of the State Council of the People's Republic of China, No. 19, 65-86.

[6] Leiter, A., Diefenbach, M.A., Doucette, J., et al. (2015) Clinical Trial Awareness: Changes over Time and Sociodemographic Disparities. Clinical Trials, 12, 215-223. https://doi.org/10.1177/1740774515571917

[7] Yang, Z., Huang, L. and Wu, Z.A. (2013) Opportunities, Challenges and Policy Suggestions for the Development of Drug Clinical Trials in China. Journal of Clinical Medicine, 22, 1619-1624.

[8] Maida, S., Costa, G.D., Rodegher, M., et al. (2014) Overcoming Recruitment Challenges in Patients with Multiple Sclerosis: Results from an Italian Survey. Clinical Trials, 11, 667-672. https://doi.org/10.1177/1740774514545248

[9] Sun, P.J., Xie, M.F. and Wang, L. (2002) Research Progress of Biologics and Small Molecule Drugs in the Treatment of Inflammatory Bowel Disease. Journal of Clinical Endocrinology, 15, 124-130.

[10] Gary, R.L., Ahva, S., Seth, A.S., et al. (2020) Burden of Crohn's Disease and Ulcerative Colitis by Age Diagnosis. Clinical Gastroenterology and Hepatology, 17, 889-897. 